Research Article

\title{
Effect of Yijin-Tang, an Oriental Traditional Formula, on Allergic Responses Using an Ovalbumin-Induced Murine Asthma Model
}

\author{
Se-Jin Lee, ${ }^{1}$ A. Yeong Lee, ${ }^{2}$ Je-Oh Lim, ${ }^{1}$ Ji Hye Lee, ${ }^{2}$ Tae-Yang Jung, ${ }^{1}$ So-Won Pak, ${ }^{1}$ \\ Woong-Il Kim, ${ }^{1}$ Yoon Soo Seo, ${ }^{2}$ Jong-Choon Kim, ${ }^{1}$ Je-Won Ko $\mathbb{D}^{,},{ }^{3}$ and In-Sik Shin $\mathbb{D}^{1}$ \\ ${ }^{1}$ College of Veterinary Medicine and BK21 FOUR Program, Chonnam National University, 77 Yongbong-ro, Buk-gu, \\ Gwangiu 61186, Republic of Korea \\ ${ }^{2}$ Herbal Medicine Resources Research Center, Korea Institute of Oriental Medicine, 111Geonjae-ro, Naju-si 58245, \\ Jeollanam-do, Republic of Korea \\ ${ }^{3}$ College of Veterinary Medicine, Chungnam National University, 99 Daehak-ro, Yuseong-gu, Daejeon 34131, Republic of Korea \\ Correspondence should be addressed to Je-Won Ko; rheoda@cnu.ac.kr and In-Sik Shin; dvmmk79@gmail.com
}

Received 14 January 2021; Revised 12 April 2021; Accepted 30 April 2021; Published 12 May 2021

Academic Editor: Man Hee Rhee

Copyright (c) 2021 Se-Jin Lee et al. This is an open access article distributed under the Creative Commons Attribution License, which permits unrestricted use, distribution, and reproduction in any medium, provided the original work is properly cited.

\begin{abstract}
Yijin-tang is an oriental traditional herb used to treat inflammatory diseases. In the present study, we investigated the protective effects of Yijin-tang water extract (YTE) using an ovalbumin- (OVA-) induced asthma model, focusing on the antioxidant and anti-inflammatory properties of the herb. BALB/c mice were intraperitoneally injected with OVA on days 0 and 14 and then challenged with OVA on days 21,22 , and 23 . The animals were orally administered YTE ( 200 and $400 \mathrm{mg} / \mathrm{kg}$ ) from days 18 to 23 , and this was found to significantly decrease airway hyperresponsiveness and release of inflammatory cells, cytokines, and OVAspecific immunoglobulin $\mathrm{E}$ in mice with asthma. In addition, YTE was associated with a marked reduction in airway inflammation and mucus production in lung tissue of mice with asthma. Furthermore, YTE suppressed the expression of matrix metalloproteinase- 9 and phosphorylation of ERK in the lungs, which in turn led to a reduction in inducible nitric oxide synthases and an elevation in reduced glutathione and heme oxygenase-1. In conclusion, YTE effectively suppressed allergic responses in mice with asthma and the effect was closely related to antioxidant and anti-inflammatory properties of the herb. Our results indicate that YTE may be a potential agent for the treatment of allergic asthma.
\end{abstract}

\section{Introduction}

Asthma is a life-threatening disease that affects human health, and its prevalence is increasing annually [1]. It is caused by various allergens, air pollutants, and chemicals, characterized by airway inflammation, airway hyperresponsiveness (AHR), and mucus overproduction [2]. Approximately 300 million people have asthma globally, and its morbidity and mortality are predicted to gradually increase [3]. The mechanisms underlying asthma are known to be very complicated and are associated with various factors such as cytokines, chemokines, and oxidative stress. These factors induce eosinophil-rich inflammation, mucus overproduction, and AHR [1]. Consequently, search has been dedicated to developing asthma treatments based on the various factors that cause asthma $[4,5]$.

Oxidative stress is closely related to the development and exacerbation of asthma [6]. An imbalance in the oxidant/ antioxidant system of an organism triggers the generation of reactive oxygen and nitrogen species (ROS and RNS, respectively), thus worsening asthmatic responses [7]. Therefore, various biological units that decrease oxidative stress are activated, particularly heme oxygenase-1 (HO-1) which acts as an important antioxidant to protect against oxidative stress [8]. Excessive ROS production upregulates HO-1 expression, which eventually decreases inducible nitric oxide synthase (iNOS) and increases antioxidant system mediators including catalase, reduced glutathione 
(GSH), and superoxide dismutase [9]. This series of events removes ROS and RNS in organisms to reduce oxidative stress, thereby alleviating asthmatic responses [8].

Yijin-tang, an oriental traditional herbal prescription, is mainly used to treat gastrointestinal diseases such as gastritis and gastric ulcers [10]. It is composed of Citrus unshiu, Glycyrrhiza uralensis, Pinellia ternate, Poria cocos, and Zingiber officinale. According to a previous study, Yijin-tang was postulated to have very strong antioxidant properties [11]. Yijin-tang has been shown to exhibit curative effects against gastric injury induced by ethanol through enhancement of antioxidant status. In addition, the crude herbs that constitute Yijin-tang have strong antioxidant properties [12-15]. Owing to this, we predicted that Yijintang would be effective in treating asthma. Moreover, a systematic review and meta-analysis reported that Yijin-tang may be used in the treatment of respiratory diseases [16]. Therefore, we explored the curative effects of Yijin-tang using an ovalbumin- (OVA-) induced asthma model, based on the antioxidant and anti-inflammatory properties of the herb.

\section{Materials and Methods}

2.1. Animals. Six-week-old BALB/c female mice were obtained from SAMTAKO (Osan, Republic of Korea). The animals were housed under normal condition $\left(22 \pm 2^{\circ} \mathrm{C}\right.$, $55 \pm 5 \% \mathrm{RH}$, and a $12 \mathrm{~h}$ night/day cycle) and received feed and water ad libitum for 5 weeks. Study approval was obtained from the Chonnam National University Institutional Animal Care and Use Committee (CNU IACUC-YBR-202023, Gwangju, Republic of Korea). Five experimental groups ( $n=5$ per group) were designed: CON (control, PBS administration), OVA (asthma model and PBS administration), DEX (asthma model and dexamethasone administration), and YTE 200 and 400 (asthma model and YTE administration (200 and $400 \mathrm{mg} / \mathrm{kg}$, respectively)).

2.2. Plants. Five Yijin-tang herbs, composed of the peel of Citrus unshiu Markovich (150 g), root of Glycyrrhiza uralensis Fischer (75 g), tuber of Pinellia ternata Breitenbach $(300 \mathrm{~g})$, sclerotium of Poria cocos Wolf (150 g), and rhizome of Zingiber officinale Roscoe (3 pieces), were purchased from Kwangmyongdang Pharmaceutical Co. (Ulsan, Republic of Korea) and analyzed for purity by Dr. Goya Choi. The individual herbs were macerated for $30 \mathrm{~min}$ and extracted by reflux in $4.8 \mathrm{~L}$ of distilled water $(\mathrm{D} \mathrm{W})$ at $100^{\circ} \mathrm{C}$ for $2 \mathrm{~h}$. The solution was filtered through chromatographic paper $(46 \times 57 \mathrm{~cm}$, Whatman Ltd., Maldstone, England) and freeze-dried to obtain $104.1 \mathrm{~g}(14.16 \%$, w/w) of Yijin-tang water extract (YTE, voucher no. YJ-1).

2.3. Chemicals. Liquiritin $(\geq 98.0 \%)$ and narirutin $(\geq 98.0 \%)$ were purchased from ChemFaces (Wuhan ChemFaces Biochemical Co., Ltd., Wuhan, China), glycyrrhizic acid ( $\geq 95.0 \%$ ) was obtained from Sigma-Aldrich (Sigma-Aldrich, Seoul, Republic of Korea), and hesperidin was purchased from Wako (Fujifilm Wako Pure Chemical Corporation,
Osaka, Japan), high-performance-liquid-chromatography(HPLC-) grade DW, acetonitrile, and methanol were obtained from Merck (Sigma-Aldrich, Darmstadt, Germany).

2.4. HPLC Analysis. Quantitative analysis of compounds containing YTE was performed using an HPLC system (Waters Corporation, Milford, MA, USA) coupled to a 2998 PDA detector, separation module (Waters e2695), micro slitter (IDEX Health and Science LLC., Oak Harbor, WA, USA), and Acquity $\mathrm{QDa}^{\mathrm{TM}}$ detector. Analytical data were processed using Empower 3 software (Waters Corporation). YTE $(121.25 \mathrm{mg})$ dissolved in DW $(10 \mathrm{~mL})$ was injected using a $0.2 \mu \mathrm{m}$ syringe filter, and constituents of YTE were separated using an Xselect HSS T3 $5 \mu \mathrm{m}$ analytical column $(250 \mathrm{~mm} \times 4.6$ i.d) (Waters Corporation) using a mobile phase flowrate of $0.8 \mathrm{~mL} / \mathrm{min}$. The mobile phase consisted of $0.05 \%$ formic acid in DW (A), methanol (B), and acetonitrile (C), and the linear gradient program was as follows: $100 \%$ A-92\% A (7\% B and 1\% C) for 0-3 min; 92\% A (7\% B and $1 \% \mathrm{C})-70 \% \mathrm{~A}(25 \% \mathrm{~B}$ and $5 \% \mathrm{C})$ for $3-16 \mathrm{~min} ; 70 \% \mathrm{~A}(25 \%$ $\mathrm{B}$ and $5 \% \mathrm{C})-60 \% \mathrm{~A}(32 \% \mathrm{~B}$ and $8 \% \mathrm{C})$ for $16-30 \mathrm{~min} ; 60 \%$ $\mathrm{A}(32 \% \mathrm{~B}$ and $8 \% \mathrm{C})$ isocratic for $30-35 \mathrm{~min} ; 60 \% \mathrm{~A}(32 \% \mathrm{~B}$ and $8 \% \mathrm{C})-55 \% \mathrm{~A}(36 \% \mathrm{~B}$ and $9 \% \mathrm{C})$ for $35-45 \mathrm{~min}$; and $55 \% \mathrm{~A}(36 \% \mathrm{~B}$ and $9 \% \mathrm{C})-0 \% \mathrm{~A}(70 \% \mathrm{~B}$ and $30 \% \mathrm{C})$ for 55-62 min. The single quadruple mass detector (QDa detector) setting was used with a probe temperature of $600^{\circ} \mathrm{C}$, an ESI capillary voltage of $0.8 \mathrm{kV}$, con voltage of $15 \mathrm{~V}, 120^{\circ} \mathrm{C}$, and a split ratio of $10: 1$. The temperatures of the autosampler and analytical column were at $10^{\circ} \mathrm{C}$ and ambient, respectively. The sample injection volume was $10 \mu \mathrm{L}$, and wavelengths were scanned from at 195-400 nm. Sample peaks were detected at $254 \mathrm{~nm}$ (for glycyrrhizic acid) and $280 \mathrm{~nm}$ (for liquiritin, narirutin, and hesperidin). The wavelengths and molecular weights of compounds found in YTE were compared against ultraviolet (UV) and mass spectrometry (MS) spectra data of respective analytical standards.

2.5. Experimental Procedure. The OVA-induced asthma model was established according to a previous study [1]. The animals were intraperitoneally injected with OVA $(20 \mu \mathrm{g}$, Sigma-Aldrich, St. Louis, MO, USA) containing aluminum hydroxide (2 mg, Sigma-Aldrich) on days 0 and 14. Subsequently, animals were administered 1\% OVA for $1 \mathrm{~h}$ via inhalation from days 21 to 23 . Dexamethasone $(1 \mathrm{mg} / \mathrm{kg})$ and YTE (200 and $400 \mathrm{mg} / \mathrm{kg}$ ) were orally administered from days 18 to 23. Airway hyperresponsiveness (AHR) was estimated on day 24 , and mice were sacrificed on day 25. Blood was collected from the caudal vena cava of each animal and then centrifuged at $200 \times \mathrm{g}$ for $20 \mathrm{~min}$ to separate out the serum.

2.6. Measurement of AHR. On day 24, we evaluated AHR by whole-body plethysmography (OCP3000, Allmedicus, Seoul, Republic of Korea). AHR was measured following aerosolization of PBS and increased concentrations of methylcholine $(10,20$, and $40 \mathrm{mg} / \mathrm{mL}$, Sigma-Aldrich) in 
PBS for $3 \mathrm{~min}$. The data are shown as the dimensionless parameter enhanced pause (Penh).

2.7. Analysis of BALF and Serum. Bronchoalveolar lavage fluid (BALF) sampling was performed as previously described [1]. Briefly, an endotracheal tube was instillated into the trachea of each animal after tracheostomy. PBS $(0.7 \mathrm{~mL})$ was administered into the left lung, and samples were transferred to the tube; this was repeated once more (to obtain $1.4 \mathrm{~mL}$ of BALF). The BALF samples were centrifuged at $200 \times \mathrm{g}$ for $10 \mathrm{~min}$, and the supernatant $(1.2 \mathrm{~mL})$ was stored at $-20^{\circ} \mathrm{C}$ for future use in evaluating cytokines. The number of total cells in BALF was evaluated using a cell counter (Countess 3 Automated Cell Counter, Thermo Fisher Scientific, Waltham, MA, USA). To perform visible inflammatory count, BALF pellets were dissolved in PBS $(200 \mu \mathrm{L})$, inflammatory cells were attached using centrifugation (Hanil, Wonju, Republic of Korea), and the slides were stained with Diff-Quik reagent (Sysmex, Kobe, Japan). The number of differential inflammatory cells in BALF pellets was obtained by applying the ratio of visible inflammatory cells to the number of total cells. BALF supernatant was evaluated for levels of interleukin- (IL-) 4, -5, and -13 using commercial ELISA kits (R\&D System, Minneapolis, MN, USA). In serum, we evaluated the levels of OVA-specific IgE by ELISA according to previous studies $[1,4]$.

2.8. Histopathology. The left lung of each animal was fixed in neutralized buffered formalin, embedded, and sections $(4 \mu \mathrm{m})$ were prepared. Sections were stained using hematoxylin and eosin (Sigma-Aldrich) and periodic acid-Schiff (IMEB Inc., San Marcos, CA, USA). Quantitative analysis of inflammatory responses and mucus production was conducted using an image analyzer (IMT i-Solution Inc., Vancouver, BC, Canada). To evaluate the expressions of HO-1 and matrix-metalloproteinase-9 (MMP-9) in the lungs, we performed immunohistochemistry (Vector Laboratories, Burlingame, CA, USA) according to previous studies [4, 17]. Anti-mouse HO-1 antibody (Abcam, Cambridge, UK) and anti-mouse MMP-9 (Abcam) were used as the primary antibodies.

2.9. Western Blotting. To investigate protein expression related to inflammation and oxidative stress, we performed western blotting according to a previous study [17]. The antibodies of phosphorylated-ERK and HO-1 were obtained from Abcam Co., and MMP-9, iNOS, GSH, and $\beta$-actin were purchased from Cell Signaling (Beverly, MA, USA). The quantitative analysis of each protein band was measured using ChemiDoc (Bio-Rad Laboratories, Hercules, CA, USA).

2.10. Statistical Analysis. All data are presented as mean\pm standard deviation. Statistical evaluation was performed using analysis of variance (ANOVA) followed by Dunnett's post hoc adjustments. $p$ values $<0.05$ and $<0.01$ were considered to be statistically significant.

\section{Results}

3.1. HPLC Analysis of YTE. The HPLC chromatogram of YTE is depicted in Figure 1. Four compounds, liquiritin (1), narirutin (2), hesperidin (3), and glycyrrhizic acid (4), were detected at approximately $32.3,35.7,38.7$, and $57.3 \mathrm{~min}$ (Figure 1(a)), respectively. Quantitation of liquiritin, narirutin, hesperidin, and glycyrrhizic acid yielded $1.56 \pm 0.00842,4.19 \pm 0.0125,3.58 \pm 0.0304 \mu \mathrm{g} / \mathrm{mg}$, and trace, respectively. UV absorption spectra of these compounds showed $\lambda_{\max }$ values of 275.9,283.1, 284.2, and $249.9 \mathrm{~nm}$, respectively. Figure 1(b) shows the total ion chromatogram and extracted ion chromatogram of the molecules. MS values $(\mathrm{m} / \mathrm{z})$ in the negative mode of the mass spectrum were confirmed as follows: liquiritin $[\mathrm{M}-\mathrm{H}]^{-}=417.10$, narirutin $[\mathrm{M}-\mathrm{H}]^{-}=579.14$, hesperidin $[\mathrm{M}-\mathrm{H}]^{-}=609.19$, and glycyrrhizic acid $[\mathrm{M}-\mathrm{H}]^{-}=821.22$.

3.2. Effect of YTE on AHR in Mice with Asthma. The OVA group exhibited a significant elevation in AHR compared with the CON group (Figure 2). In contrast, the DEX group showed a markedly decreased AHR in comparison to the OVA group. Similarly, YTE groups also showed a notable reduction in AHR in comparison to the OVA group; the decrease was more evident in the YTE-400 group.

\subsection{Effect of YTE on Accumulation of Inflammatory Cells in} BALF of Mice with Asthma. The OVA group showed a marked elevation in inflammatory cell count in comparison to the CON group (Figure 3). Particularly, the elevation in BALF eosinophil count was detected in the OVA group. In contrast, the DEX group exhibited a noticeable reduction in BALF inflammatory cell count in comparison to the OVA group. Similar to the results of the DEX group, YTE groups showed a notable reduction in inflammatory cell counts in comparison to the OVA group, however, the decline being more evident in the YTE-400 group.

3.4. Effect of YTE on Cytokine Levels and OVA-Specific IgE in Mice with Asthma. The OVA group showed a notable elevation in BALF IL-4 level in comparison to the CON group (Figure 4(a)). In contrast, the DEX group significantly decreased IL-4 level as compared to the OVA group. In addition, the YTE groups exhibited a significant reduction in BALF IL-4 levels in comparison to the OVA group in a dosedependent manner. Consistent with the results of IL-4, the levels of IL-5 and - 13 markedly decreased in the YTE groups compared to those observed in the OVA group (Figures 4(b) and 4(c), respectively). The levels of OVA-specific IgE were markedly elevated in the OVA group in comparison with those seen in the CON group (Figure 4(d)). Conversely, the YTE groups exhibited substantially reduced OVA-specific IgE level compared with the OVA group. The YTE-400 


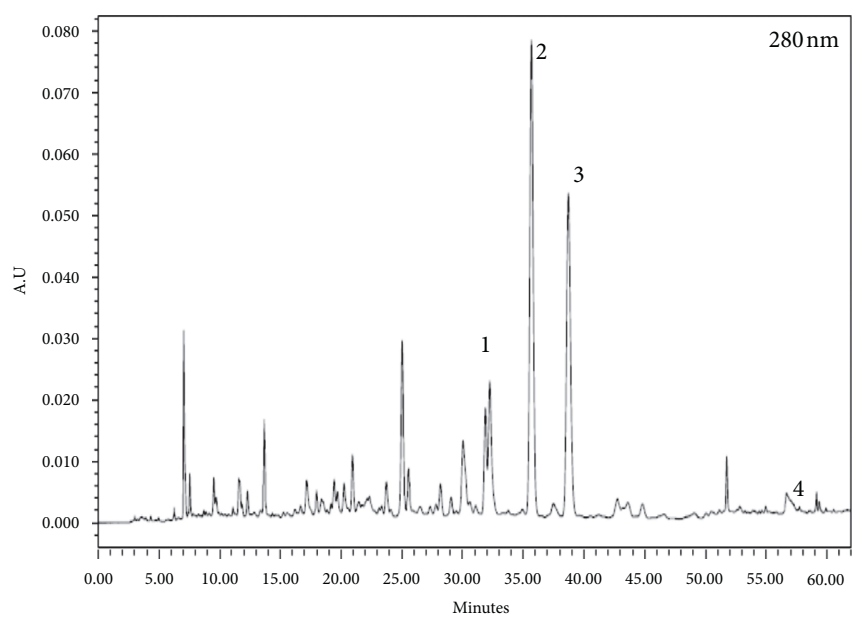

(a)

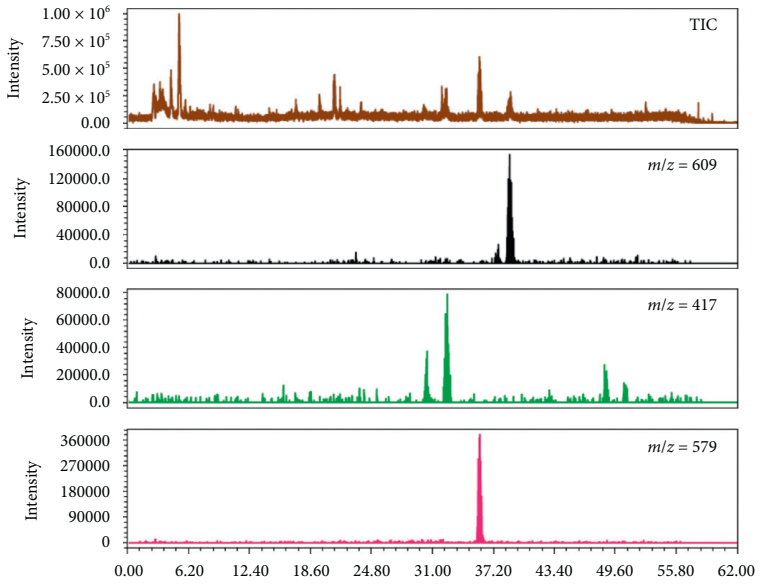

(b)

FIGURE 1: Chromatogram of YTE at $280 \mathrm{~nm}$ (a) and total ion chromatogram and selected ion chromatogram (b).

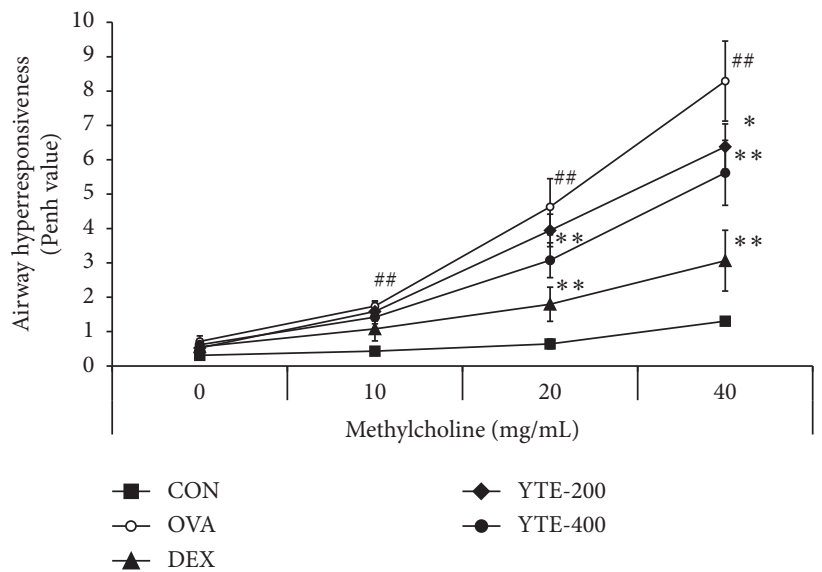

FIgURE 2: Effect of YTE on AHR in an OVA-induced asthma mouse model. AHR was regarded as a crucial clinical sign exhibiting respiratory contraction in asthma and evaluated using whole-body plethysmograph. CON, PBS administration; OVA, asthma model and PBS administration; DEX, asthma model and dexamethasone administration; YTE-200 and -400, OVA asthma model and YTE administration. ${ }^{\# \#} p<0.01$ vs CON; ${ }^{* * *} p<0.05$ and 0.01 vs. OVA, respectively.

group showed a notable decline in OVA-specific IgE levels in comparison to the OVA group.

\subsection{Effect of YTE on Inflammation and Mucus Production in} Mice with Asthma. The OVA group exhibited a marked accumulation of inflammatory cells in lung tissue in comparison to the CON group (Figures 5(a) and 5(b)). However, the DEX group showed a notable reduction in airway inflammation in comparison to the OVA group. Consistent with results of the DEX group, YTE groups showed significant decreases in accumulation of inflammatory cell in lung tissue in comparison to the OVA group in a dosedependent manner. Furthermore, the OVA group showed a notable elevation in mucus production compared with the CON group (Figures 5(a) and 5(c)). On the contrary, the DEX group exhibited significantly decreased mucus production compared with the OVA group. The YTE groups showed decreased mucus production in comparison to the
OVA group. In particular, the YTE-400 group showed a significant reduction in mucus production in comparison to the OVA group.

3.6. Effect of YTE on Antioxidant Signaling in Mice with Asthma. The OVA group exhibited a marked increase in the expression of iNOS expression in comparison to the CON group (Figures 6(a) and 6(b)). However, the YTE groups showed a notable reduction in iNOS expression in comparison to the OVA group. The expression levels of GSH remarkably decreased in the OVA group in comparison to those observed in the CON group, whereas a significant increase was observed in YTE groups compared with the OVA group. In addition, the OVA group exhibited an elevation in the expression of HO-1 in comparison to the CON group (Figures 6(a) and 6(c)). Moreover, the expression levels of HO-1 were significantly increased in YTE groups compared to the OVA group. 


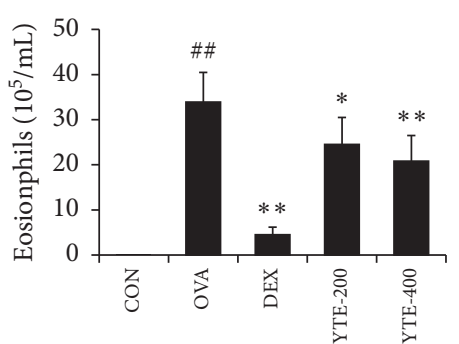

(a)

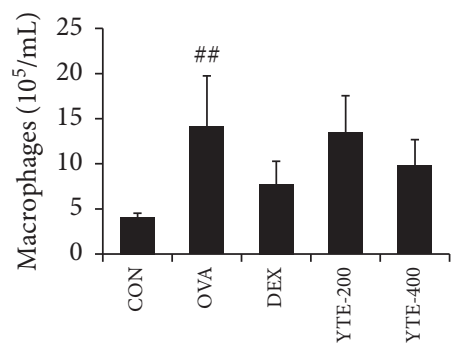

(b)

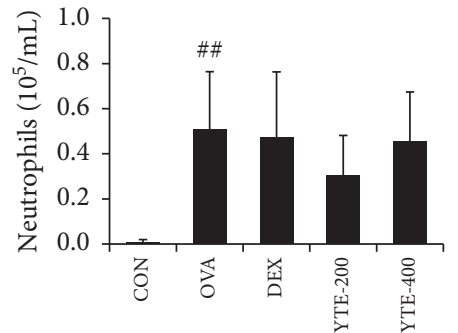

(c)

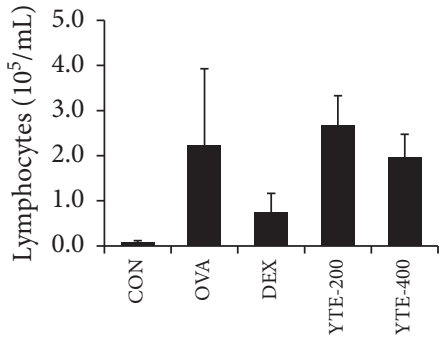

(d)

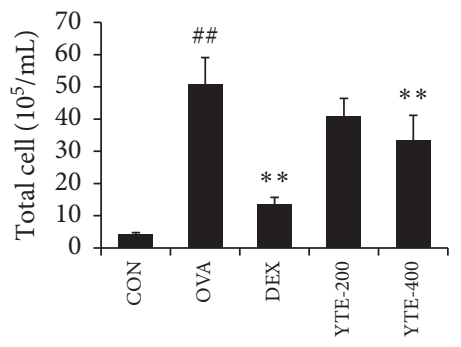

(e)

FIGURE 3: Effect of YTE on accumulation of inflammatory cells in BALF of mice with asthma. Inflammatory cell count was markedly increased in the development of asthma. (a) eosinophils, (b) macrophages, (c) neutrophils, (d) lymphocytes, and (e) total cells. CON, PBS administration; OVA, asthma model and PBS administration; DEX: asthma model and dexamethasone administration; YTE-200 and -400, asthma model and YTE administration. ${ }^{\# \#} p<0.01$ vs. CON; ${ }^{* * *} p<0.05$ and 0.01 vs. OVA, respectively.

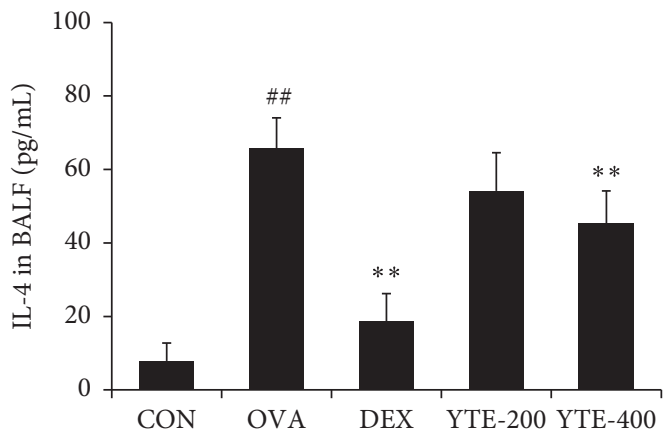

(a)

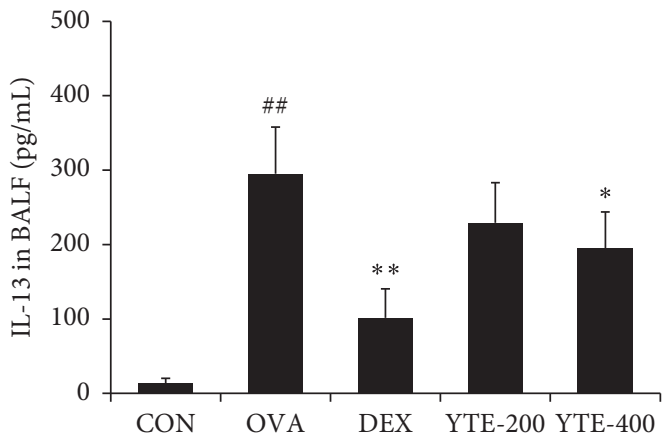

(c)

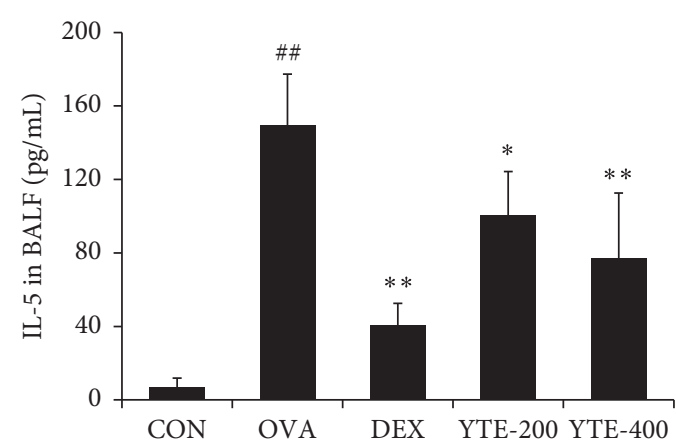

(b)

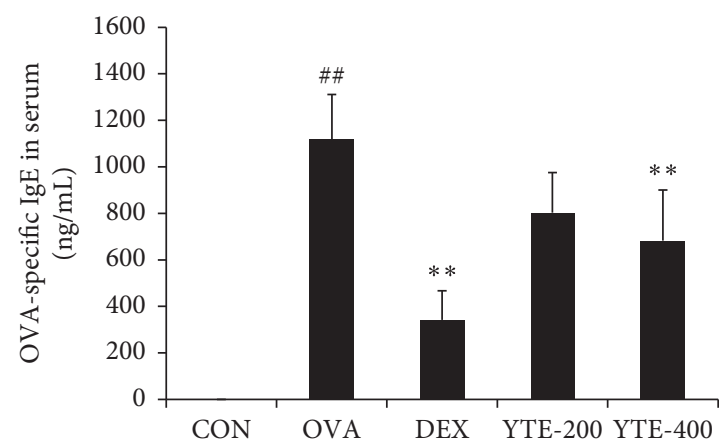

(d)

FIgURE 4: Effect of YTE on the levels of cytokines and OVA-specific IgE in mice with asthma. The cytokines were associated with the allergic responses such as eosinophilia, AHR, and IgE production. (a) IL-4, (b) IL-5, (c) IL-13, and (d) OVA-specific IgE. CON, PBS administration; OVA, asthma model and PBS administration; DEX, asthma model and dexamethasone administration; YTE-200 and -400, asthma model and YTE administration. ${ }^{\# \#} p<0.01$ vs. CON; ${ }^{* * *} p<0.05$ and 0.01 vs. OVA, respectively. 


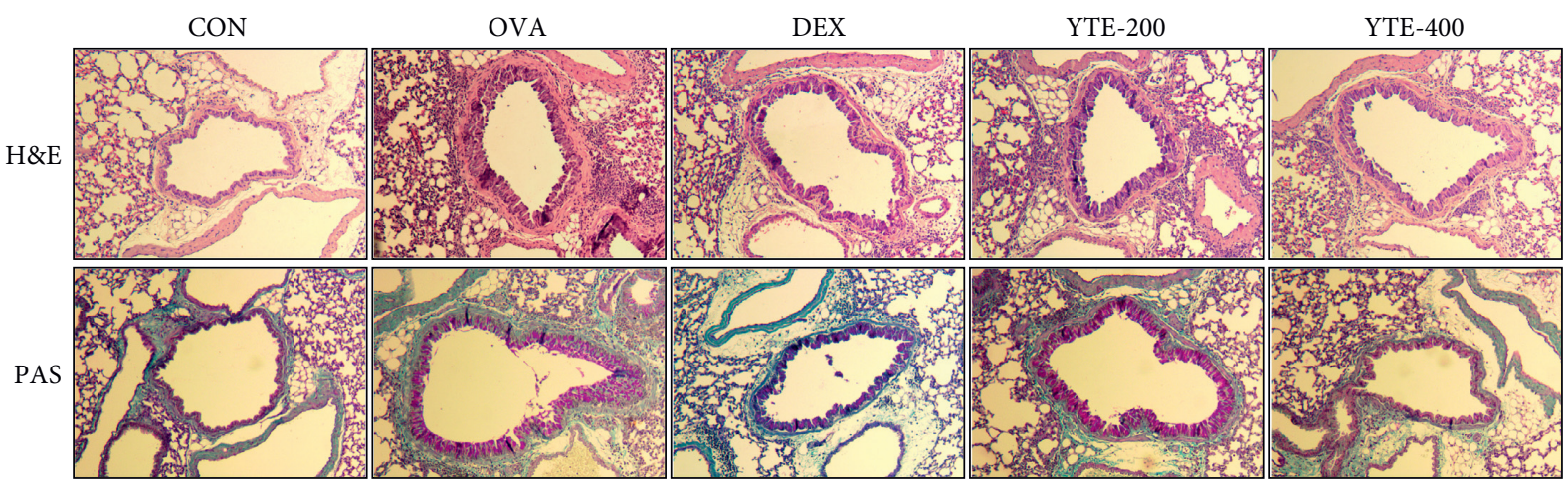

(a)

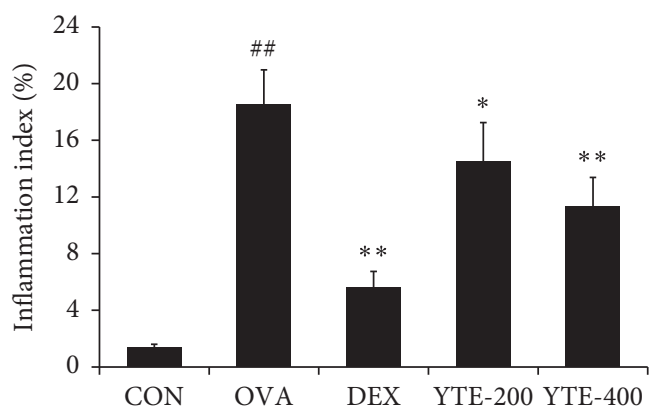

(b)

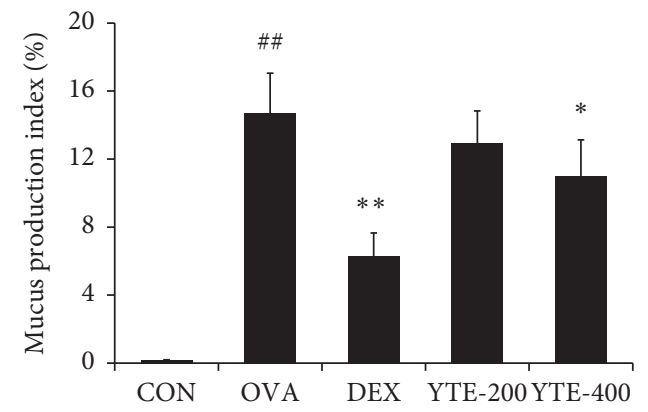

(c)

FIGURE 5: Effect of YTE on airway inflammation and mucus production in mice with asthma. Allergic asthma was featured as airway inflammation and mucus production in respiratory tract. (a) Histological figure of lung tissue, (b) inflammation Index, and (c) mucus production index. CON, PBS administration; OVA, asthma model and PBS administration; DEX, asthma model and dexamethasone administration; YTE-200 and -400, asthma model and YTE administration. ${ }^{\# \#} p<0.01$ vs. CON; ${ }^{* * *} p<0.05$ and 0.01 vs. OVA, respectively.

3.7. Effect of YTE on Inflammatory Signaling in Mice with Asthma. The OVA group exhibited a notable elevation in the expression levels of MMP-9 compared to the CON group (Figures $7(\mathrm{a})-7(\mathrm{c}))$. In contrast, the YTE groups showed a notable reduction expression levels of in MMP-9 in comparison to the OVA group in a dose-dependent manner. Furthermore, the OVA group markedly increased the phosphorylation of ERK in comparison to the CON group. However, the YTE groups, in a dose-dependent manner, significantly declined the phosphorylation of ERK in comparison to the OVA group.

\section{Discussion}

Asthma is a chronic inflammatory disorder with a high global incidence and is currently considered a serious life- threatening condition in those with respiratory diseases caused by fine dust, bacteria, and viruses $[2,18,19]$. In the present study, we explored the therapeutic effects of YTE on asthmatic responses using an OVA-induced asthma model. The administration of YTE significantly suppressed the elevation of AHR, cytokines, eosinophils, and OVA-specific IgE in mice with asthma, in addition to reducing airway inflammation and mucus production in the lungs tissue. Furthermore, the administration of YTE significantly decreased the phosphorylation of ERK and the expression of MMP-9 in mice, resulting in an elevation in the expression levels of HO-1 and GSH and a reduction in iNOS.

Eosinophilia is an important feature in the advancement of asthma [20]. Eosinophils are regarded as crucial biomarkers of allergic response and are closely related to inflammatory cytokines, including IL-4, -5, and -13, during the 


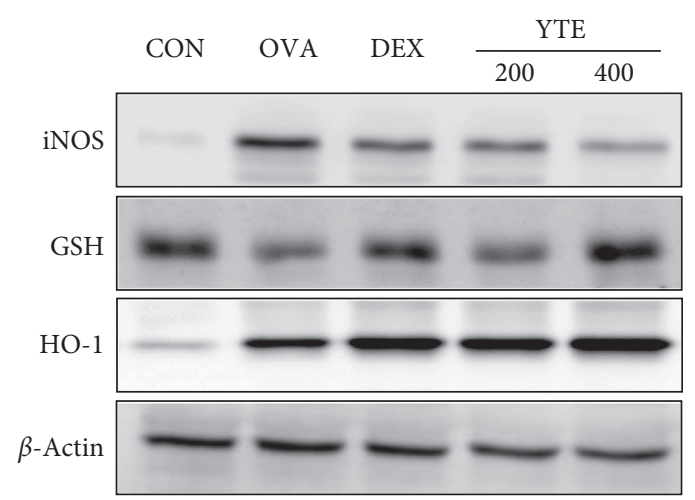

(a)

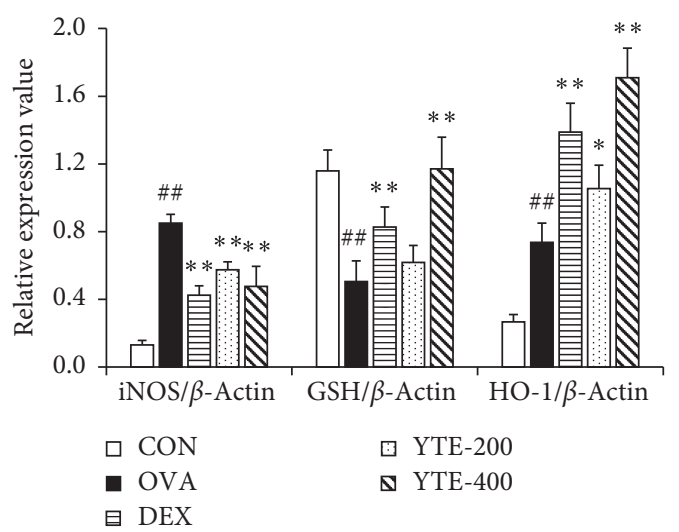

(b)

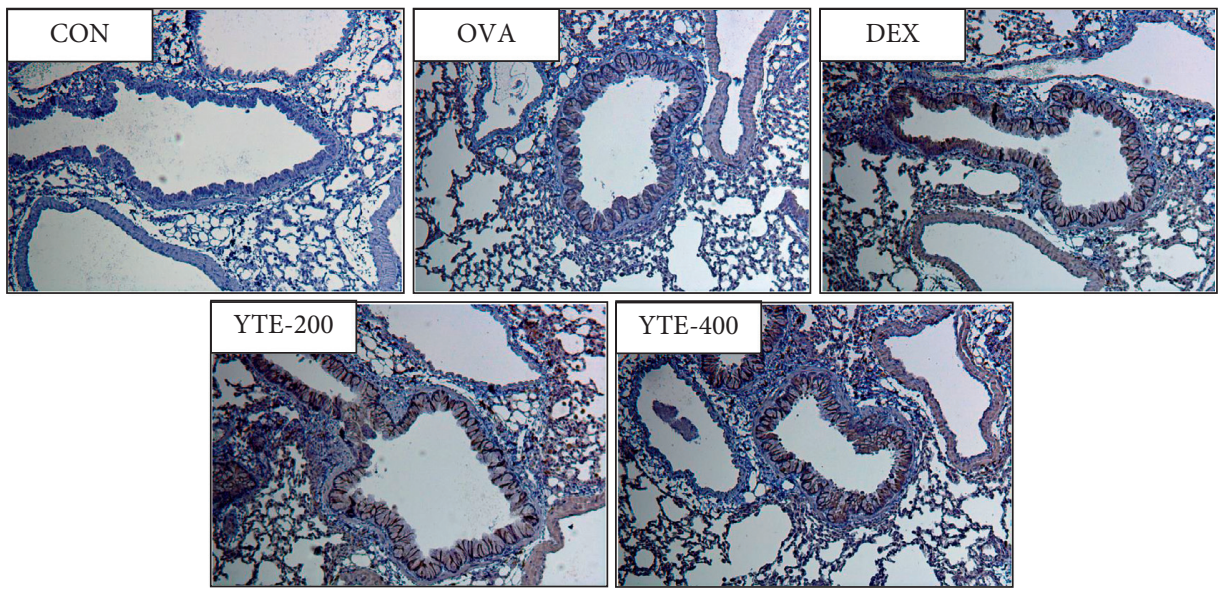

(c)

FIGURE 6: Effect of YTE on antioxidative signaling in mice with asthma. HO-1 acts as an antioxidant enzyme and decreases asthmatic responses. (a) Protein expression on gel, (b) relative expression value, and (c) representative figure for lung tissue following immunohistochemistry (IHC) of HO-1. CON, PBS administration; OVA, asthma model and PBS administration; DEX, asthma model and dexamethasone administration; YTE-200 and -400 , asthma model and YTE administration. ${ }^{\# \#} p<0.01$ vs. CON; ${ }^{* * *} p<0.05$ and 0.01 vs. OVA, respectively.

progression of asthma [21]. Cytokines not only trigger not the release of eosinophils into damaged lesions but also aid in the maturation and activation of eosinophils [20]. The activation of eosinophils triggers the releases of various stimulating factors such as ROS, RNS, cytokines, chemokines, and growth factors in granules, eventually triggering AHR, airway inflammation, and mucus overproduction [22]. In this study, administration of YTE reduced the production of inflammatory cytokines, which subsequently decreased the infiltration of inflammatory cells into lung tissue and the production of OVA-specific IgE. Through this cascade, YTE caused a reduction in AHR and inflammatory responses in lung tissue. These results suggest that YTE had a therapeutic effect on the allergic response that occurred in OVA-sensitized mice.

Airway inflammation is caused by various proinflammatory factors. In particular, MMP-9 is a critical mediator in the release of inflammatory cells into inflammatory lesions [23]. MMP-9 promotes airway remodeling by facilitating the degradation of various substrates that make up normal alveolar tissue and induces the release of inflammatory cells into damaged lesions, which ultimately aggravates airway inflammation [24]. The expression of MMP-9 is associated with the phosphorylation of ERK. ERK is a mitogen-activated protein kinase MAPK and is regarded as a critical factor in inflammatory responses; its phosphorylation markedly increases the expression levels of MMP-9 during the progression of asthma [25]. According to a previous study [26], an increase in RK phosphorylation caused an upregulation of MMP-9 expression, which elevated the levels of various inflammatory mediators. In this study, administration of YTE decreased the expression levels of MMP-9 in mice with asthma, as well as reducing ERK phosphorylation. These results suggest that the observed anti-inflammatory properties of YTE could be due to the downregulation of ERK phosphorylation and expression of MMP-9.

Excessive production of ROS is a crucial factor in the progression of asthma [27]. Airway inflammation was found to cause the elevation of ROS production in airway epithelia, eosinophils, macrophages, and neutrophils, which were related to the damage of various biological molecules [28]. In 


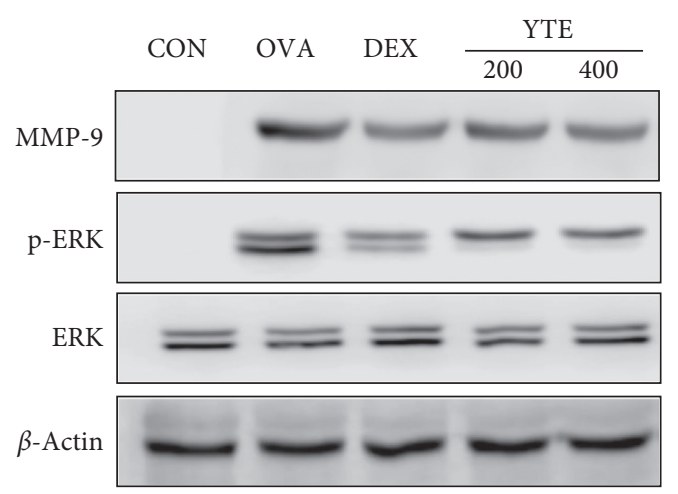

(a)

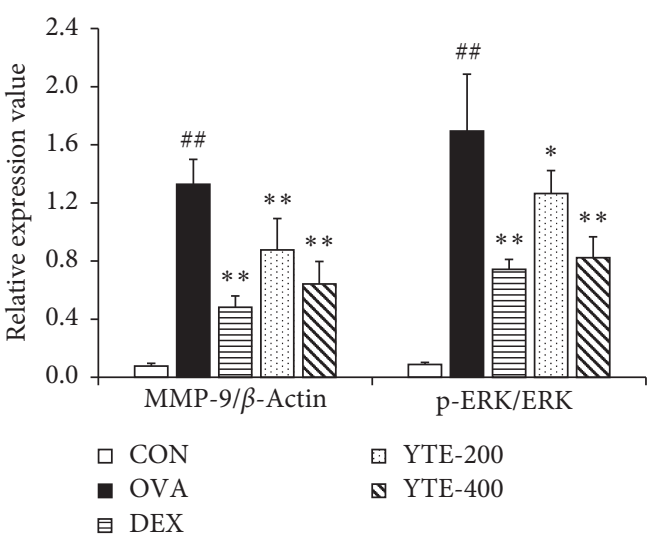

(b)

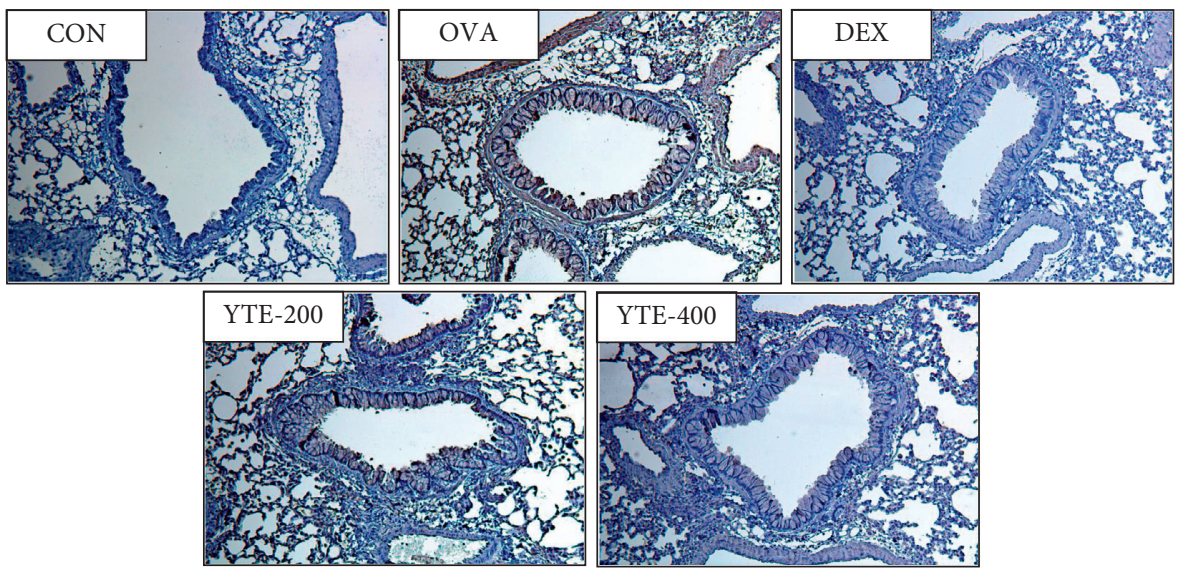

(c)

FIGURE 7: Effect of YTE on the inflammatory signaling in mice with asthma. MMP-9 and ERK were associated with the development of inflammatory response in asthma. (a) Protein expression on gel, (b) relative expression value, and (c) representative figure for lung tissue following IHC of MMP-9. CON, PBS administration; OVA, asthma model and PBS administration; DEX, asthma model and dexamethasone administration; YTE-200 and -400 , asthma model and YTE administration. ${ }^{\# \#} p<0.01 \mathrm{vs.} \mathrm{CON;}{ }^{* * *} p<0.05$ and 0.01 vs. OVA, respectively.

addition, cytokines were found to be associated with the production of ROS [29]. Furthermore, accumulation of ROS was shown to induce AHR by decreasing the function of $\beta$-adrenergic receptors, releasing histamine from mast cells, and increasing mucus secretion from goblet cells [27]. Moreover, ROS was reported to cause airway epithelial barrier damage, increasing the permeability of inflammatory cells and mediators, resulting in aggravation of airway inflammation $[27,30]$. On the contrary, numerous antioxidant biological molecules or systems against oxidative stress exist. HO-1 is part of an antioxidant system that reduces oxidative stress by elevating antioxidant materials such as GSH, resulting in the attenuation of inflammatory responses $[31,32]$. Therefore, upregulation of HO-1 expression causes an increase in antioxidant status in organisms, thereby reducing oxidative stress and eventually alleviating airway inflammation, a major feature of asthma, via reducing the production of inflammatory mediators [33, 34]. In this study, administration of YTE resulted in a significant elevation in expression levels of $\mathrm{HO}-1$ in asthma-induced mice, which was accompanied by an increase and decrease in expression levels of GSH and iNOS, respectively. These results showed that YTE reduced asthmatic responses in mice through the upregulation of HO-1 expression.

Antiasthmatic effects of YTE observed in this study were supported by various previous studies $[11,12,16]$. The crude herbs that make up YTE have been shown to have antiinflammatory and antioxidant properties in various research studies [12-15]. Furthermore, we characterized the active components in YTE using HPLC. Liquiritin, narirutin, hesperidin, and glycyrrhizic acid were detected as the active constituents of YTE. These compounds have been reported to possess anti-inflammatory and antioxidant properties in various studies in the literature [35-38]. Therefore, it is thought that the antiasthmatic effects observed from the use of YTE are closely associated with the aforementioned active moieties.

This study showed that YTE possesses effects that support a favorable prognosis of asthma. However, it is difficult to single out which constituents of YTE exhibit effects that manage the symptoms of asthma. Therefore, it is vital to conduct more experiments, including in vitro and in vivo 
studies for each YTE constituent, to determine the active compound(s) with antiasthmatic effect. Moreover, the antiinflammatory mechanism of YTE identified in this study needs to be clearly demonstrated through genetically engineered mice or cells.

\section{Conclusions}

Overall, YTE decreased asthmatic responses including AHR and release of inflammatory cells, inflammatory cytokines, and OVA-specific IgE levels in asthma-induced mice. These properties of YTE were related to the inhibition of ERK phosphorylation and upregulation of HO-1 expression. Therefore, the study findings suggest that YTE has the ability to be used as a potential antiasthmatic agent.

\section{Data Availability}

The data used to support the findings of this study are available from the corresponding author upon request.

\section{Conflicts of Interest}

The authors report no conflicts of interest.

\section{Authors' Contributions}

Se-Jin Lee contributed equally to this work.

\section{Acknowledgments}

This research was supported by projects entitled "University Innovation Support Project of Chungnam National University" (grant no. 2020-2021).

\section{References}

[1] N. R. Shin, S. H. Park, J. W. Ko et al., "Lobeglitazone attenuates airway inflammation and mucus hypersecretion in a murine model of ovalbumin-induced asthma," Frontiers in Pharmacology, vol. 9, p. 906, 2018.

[2] N. Greenberg, R. S. Carel, E. Derazne et al., "Different effects of long-term exposures to $\mathrm{SO}_{2}$ and $\mathrm{NO}_{2}$ air pollutants on asthma severity in young adults," Journal of Toxicology and Environmental Health, Part A, vol. 79, no. 8, pp. 342-351, 2016.

[3] S. C. Dharmage, J. L. Perret, and A. Custovic, "Epidemiology of asthma in children and adults," Frontiers in Pediatrics, vol. 7, p. 246, 2019.

[4] J.-W. Ko, N.-R. Shin, S.-H. Park et al., "Genipin inhibits allergic responses in ovalbumin-induced asthmatic mice," International Immunopharmacology, vol. 53, pp. 49-55, 2017.

[5] N. R. Shin, H. W. Ryu, J. W. Ko et al., "Artemisia argyi attenuates airway inflammation in ovalbumin-induced asthmatic animals," Journal of Ethnopharmacology, vol. 209, pp. 08-115, 2017.

[6] M. Xu, L. Wang, M. Wang et al., "Mitochondrial ROS and NLRP3 inflammasome in acute ozone-induced murine model of airway inflammation and bronchial hyperresponsiveness," Free Radical Research, vol. 53, no. 7, pp. 780-790, 2019.

[7] H. Cui, J. Huang, M. Lu et al., "Antagonistic effect of vitamin E on $\mathrm{nAl}_{2} \mathrm{O}_{3}$-induced exacerbation of Th2 and Th17-mediated allergic asthma via oxidative stress," Environmental Pollution, vol. 252, pp. 1519-1531, 2019.

[8] B. W. Lee, J. H. Ha, H. G. Shin et al., "Lindera obtusiloba attenuates oxidative stress and airway inflammation in a murine model of ovalbumin-challenged asthma Antioxidants," Basel), vol. 9, no. 7, 2020.

[9] Y. Pan, W. Li, Y. Feng, J. Xu, and H. Cao, "Edaravone attenuates experimental asthma in mice through induction of HO-1 and the Keap1/Nrf2 pathway," Experimental and Therapeutic Medicine, vol. 19, no. 2, 2020.

[10] A. Y. Lee, W. Park, T.-W. Kang, M. H. Cha, and J. M. Chun, "Network pharmacology-based prediction of active compounds and molecular targets in Yijin-Tang acting on hyperlipidaemia and atherosclerosis," Journal of Ethnopharmacology, vol. 221, pp. 151-159, 2018.

[11] I.-S. Shin, M.-Y. Lee, C.-S. Seo, H.-S. Lim, H.-K. Ha, and H.-K. Shin, "Yijin-tang, an oriental herbal formula reduces ethanol-induced acute gastric injury in rats," Journal of the Korean Society for Applied Biological Chemistry, vol. 55, no. 2, pp. 197-204, 2012.

[12] D.-S. Kim and S.-B. Lim, "Semi-continuous subcritical water extraction of flavonoids from citrus unshiu peel: their antioxidant and enzyme inhibitory activities," Antioxidants, vol. 9, no. 5, p. 360, 2020.

[13] M. Wang, W. Yang, X. Liu et al., "Two new compounds with Nrf2 inducing activity from glycyrrhiza uralensis," Natural Product Research, vol. 30, pp. 1-8, 2020.

[14] J. Liu, J. Yu, and X. Peng, "Poria cocos polysaccharides alleviates chronic nonbacterial prostatitis by preventing oxidative stress, regulating hormone production, modifying gut microbiota, and remodeling the DNA methylome," Journal of Agricultural and Food Chemistry, vol. 68, no. 45, pp. 1266112670, 2020.

[15] Q.-Q. Mao, X.-Y. Xu, S.-Y. Cao et al., "Bioactive compounds and bioactivities of ginger (zingiber officinale Roscoe)," Foods, vol. 8, no. 6, p. 185, 2019.

[16] L. Deng, X. Zhang, Y. Dong et al., "Erchen decoction combined with Sanziyanqin decoction for chronic obstructive pulmonary disease: a protocol for systematic review and metaanalysis Medicine," Baltimore, vol. 99, no. 40, Article ID e22315, 2020.

[17] T. Y. Jung, A. Y. Lee, J. H. Song et al., "Scrophularia koraiensis Nakai attenuates allergic airway inflammation via suppression of NF-kB and enhancement of Nrf2/HO-1 signaling Antioxidants," Basel, vol. 9, no. 2, p. 99, 2020.

[18] J. Zhang, C. C. Fulgar, T. Mar et al., "TH17-Induced neutrophils enhance the pulmonary allergic response following $\mathrm{BALB} / \mathrm{c}$ exposure to house dust mite allergen and fine particulate matter from California and China," Toxicological Sciences, vol. 164, no. 2, pp. 627-643, 2018.

[19] S. C. Lee, K. J. Son, C. H. Han, J. Y. Jung, and S. C. Park, "Impact of comorbid asthma on severity of coronavirus disease (COVID-19)," Scientific Reports, vol. 10, no. 1, p. 21805, 2020.

[20] I. S. Shin, J. W. Park, N. R. Shin et al., "Melatonin inhibits MUC5AC production via suppression of MAPK signaling in human airway epithelial cells," Journal of Pineal Research, vol. 56, no. 4, 2014.

[21] P. J. Barnes, "Cellular and molecular mechanisms of asthma and COPD," Clinical Science Lond, vol. 131, no. 13, pp. 1541-1558, 2017.

[22] S.-M. Kim, H. W. Ryu, O.-K. Kwon et al., "Callicarpa japonica Thunb. ameliorates allergic airway inflammation by suppressing NF- $\kappa \mathrm{B}$ activation and upregulating $\mathrm{HO}-1$ 
expression," Journal of Ethnopharmacology, vol. 267, Article ID 113523, 2021.

[23] I.-S. Shin, J.-W. Park, N.-R. Shin et al., "Melatonin reduces airway inflammation in ovalbumin-induced asthma," Immunobiology, vol. 219, no. 12, pp. 901-908, 2014.

[24] K. Grzela, M. Litwiniuk, W. Zagorska, and T. Grzela, "Airway remodeling in chronic obstructive pulmonary disease and asthma: the role of matrix metalloproteinase-9," Archivum Immunologiae et Therapiae Experimentalis, vol. 64, no. 1, pp. 47-55, 2016.

[25] J.-M. Hong, O.-K. Kwon, I.-S. Shin et al., “Anti-inflammatory activities of Physalis alkekengi var. franchetii extract through the inhibition of MMP-9 and AP-1 activation," Immunobiology, vol. 220, no. 1, pp. 1-9, 2015.

[26] G. J. Huang, S. S. Huang, and J. S. Deng, “Anti-inflammatory activities of inotilone from Phellinus linteus through the inhibition of MMP-9, NF-kB, and MAPK activation in vitro and in vivo," PLoS One, vol. 7, no. 5, Article ID e35922, 2012.

[27] J. Checa and J. M. Aran, "Airway redox homeostasis and inflammation gone awry: from molecular pathogenesis to emerging therapeutics in respiratory pathology," International Journal of Molecular Sciences, vol. 21, no. 23, p. 9317, 2020.

[28] J. Qu, Y. Li, W. Zhong, P. Gao, and C. Hu, "Recent developments in the role of reactive oxygen species in allergic asthma," Journal of Thoracic Disease, vol. 9, no. 1, pp. E32-E43, 2017.

[29] B. N. Lambrecht, H. Hammad, and J. V. Fahy, "The cytokines of asthma," Immunity, vol. 50, no. 4, pp. 975-991, 2019.

[30] P. He, M. A. H. Talukder, and F. Gao, "Oxidative stress and microvessel barrier dysfunction," Frontiers in Physiology, vol. 11, p. 472, 2020.

[31] I.-C. Lee, S.-H. Kim, H.-S. Baek et al., "The involvement of $\mathrm{Nrf} 2$ in the protective effects of diallyl disulfide on carbon tetrachloride-induced hepatic oxidative damage and inflammatory response in rats," Food and Chemical Toxicology, vol. 63, pp. 174-185, 2014.

[32] J. A. Lee, M. Y. Lee, I. S. Shin et al., "Anti-inflammatory effects of Amomum compactum on RAW 264.7 cells via induction of heme oxygenase-1," Archives of Pharmacal Research, vol. 35, no. 4, 2012.

[33] W. Y. Jeon, H. K. Shin, I. S. Shin, S. K. Kim, and M. Y. Lee, "Soshiho-tang water extract inhibits ovalbumin-induced airway inflammation via the regulation of heme oxygenase-1," BMC Complementary and Alternative Medicine, vol. 15, p. 329, 2015.

[34] I.-S. Shin, J. Hong, C.-M. Jeon et al., "Diallyl-disulfide, an organosulfur compound of garlic, attenuates airway inflammation via activation of the Nrf-2/HO-1 pathway and NFkappaB suppression," Food and Chemical Toxicology, vol. 62, pp. 506-513, 2013.

[35] X. Q. Li, L. M. Cai, J. Liu et al., "Liquiritin suppresses UVBinduced skin injury through prevention of inflammation, oxidative stress and apoptosis through the TLR4/MyD88/NFkappaB and MAPK/caspase signaling pathways," International Journal of Molecular Medicine, vol. 42, no. 3, pp. 1445-1459, 2018.

[36] S. K. Ha, H.-Y. Park, H. Eom, Y. Kim, and I. Choi, "Narirutin fraction from citrus peels attenuates LPS-stimulated inflammatory response through inhibition of NF- $\kappa \mathrm{B}$ and MAPKs activation," Food and Chemical Toxicology, vol. 50, no. 10, pp. 3498-3504, 2012.

[37] S. A. Adefegha, N. B. Bottari, D. B. Leal, C. M. de Andrade, and M. R. Schetinger, "Interferon gamma/interleukin-4 modulation, anti-inflammatory and antioxidant effects of hesperidin in complete Freund's adjuvant (CFA)-induced arthritis model of rats," Immunopharmacology and Immunotoxicology, vol. 42, no. 5, pp. 509-520, 2020.

[38] R.-H. Jiang, J.-J. Xu, D.-C. Zhu et al., "Glycyrrhizin inhibits osteoarthritis development through suppressing the PI3K/ AKT/NF- $\kappa$ B signaling pathway in vivo and in vitro," Food \& Function, vol. 11, no. 3, pp. 2126-2136, 2020. 